\title{
Ablation, Thermal Response, and Chemistry Program for Analysis of Thermal Protection Systems
}

\author{
F.S. Milos ${ }^{*}$ and Y.-K. Chen ${ }^{\dagger}$ \\ NASA Ames Research Center, Moffett Field, CA 94035-1000
}

\begin{abstract}
In previous work, the authors documented the Multicomponent Ablation Thermochemistry (MAT) and Fully Implicit Ablation and Thermal response (FIAT) programs. In this work, key features from MAT and FIAT were combined to create the new Fully Implicit Ablation, Thermal response, and Chemistry (FIATC) program. FIATC is fully compatible with FIAT (version 2.5) but has expanded capabilities to compute the multispecies surface chemistry and ablation rate as part of the surface energy balance. This new methodology eliminates $B^{\prime}$ tables, provides blown species fractions as a function of time, and enables calculations that would otherwise be impractical (e.g. 4+ dimensional tables) such as pyrolysis and ablation with kinetic rates or unequal diffusion coefficients. Equations and solution procedures are presented, then representative calculations of equilibrium and finite-rate ablation in flight and ground-test environments are discussed.
\end{abstract}

Nomenclature

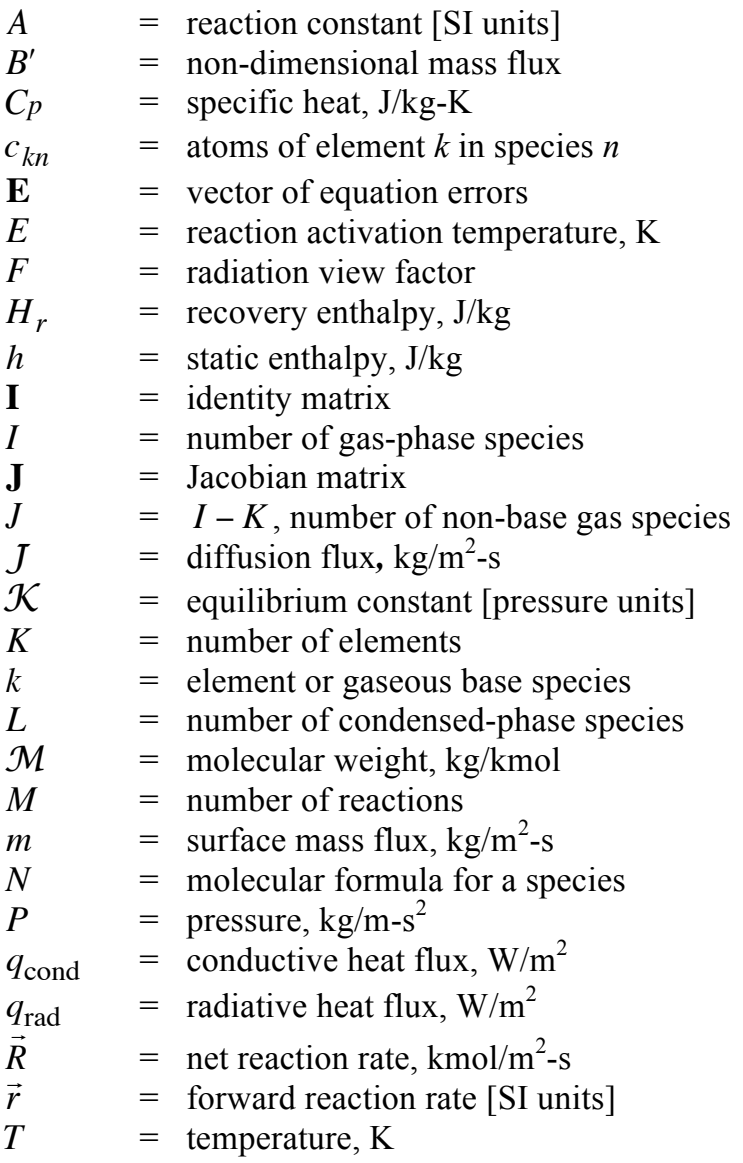
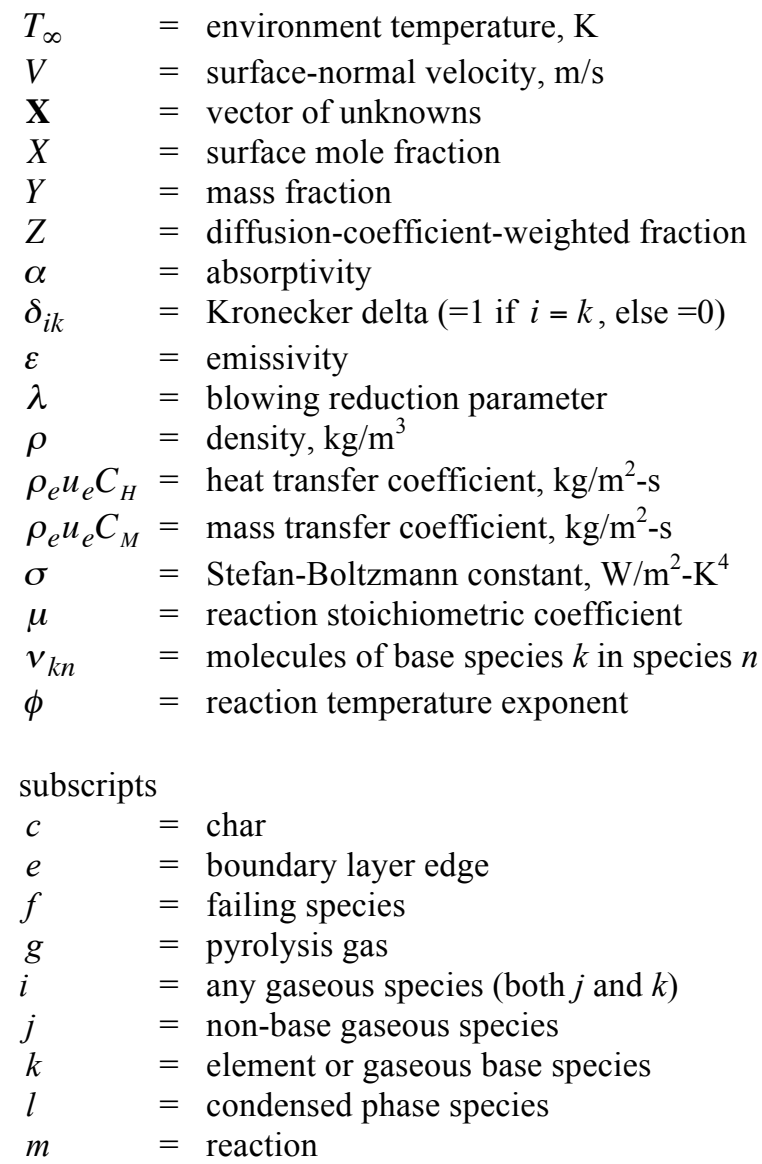

\footnotetext{
* Aerospace Engineer, Thermal Protection Materials Branch, MS 234-1. Senior Member AIAA.

${ }^{\dagger}$ Aerospace Engineer, Aerothermodynamics Branch, MS 234-1. Member AIAA.
} 


$\begin{aligned} n & =\text { all species (both } i \text { and } l \text { ) } \\ w & =\text { surface value } \\ 1 & =\text { unblown value }\end{aligned}$

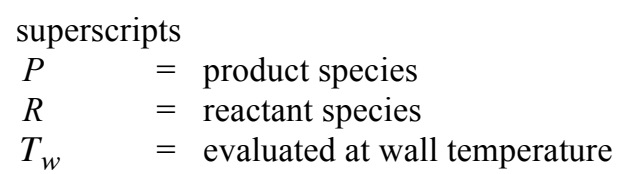

\section{Introduction}

TN existing analysis codes for thermal protection system (TPS) materials, the ablation chemistry is modeled (at $\mathbf{I}_{\text {best) }}$ using tables that are created in advance for specified compositions of material and of atmospheric gas. In the past, thermochemical ablation tables for specific gas environments and thermal protection materials were generated using codes such as ACE or MAT. ${ }^{1,2}$ Each line of the table represents a minimal amount of information from a solution of the multispecies ablation chemistry with various applicable constraints. These tables are input and interpolated by TPS analysis codes such as CMA or FIAT ${ }^{3,4}$ that solve the time-dependent material response, but the detailed chemical information such as blown species concentrations is lost.

This methodology is subject to errors and uncertainties from table interpolations. Additionally, for complex problems such as pyrolysis and ablation with kinetically-controlled rates or unequal diffusion coefficients, it is impractical to create and to interpolate tables with four or more independent variables. Furthermore, for the same materials, different tables are needed for each atmospheric or ground-test environment to be analyzed. This requirement is cumbersome and inconvenient. For example, the Orion and MSL Projects tested Phenolic Impregnated Carbon Ablator (PICA) in various arcjet environments, ${ }^{5}$ and analyses were performed for vehicles and trajectories for both Earth and Mars. To perform analyses for this collection of test and flight environments, ablation tables were generated for 16 different mixtures of nitrogen, oxygen, argon, and carbon dioxide - which resulted in over 90,000 lines of input for the PICA material.

In this work, we extensively revised both the MAT chemistry code and the FIAT ablation and thermal response code to enable the integration of the two codes. The multispecies chemistry is embedded within the surface energy balance (SEB) boundary condition of the new Fully Implicit Ablation, Thermal response, and Chemistry (FIATC) code. In FIATC the ablation and gas species blowing rates are calculated dynamically as part of the material response solution, without using $B^{\prime}$ tables. ${ }^{\ddagger}$ Incorporation of this chemistry package within FIATC enables the calculation of more complex problems, as mentioned previously, and several future upgrades are planned including ablators with graded composition, pyrolysis gas chemistry and coking, and stacked pyrolyzing ablators. For the PICA example given above, the input is under 150 lines of data and independent of the gas composition.

The purpose of this work is to document the equations and solution procedures in FIATC, then to present representative calculations of ablation in ground-test and flight environments. [In the following sections we define the general equations with material fail, unequal diffusion coefficients, and unequal heat and mass transfer coefficients. We have not yet implemented all of these models, but plan to do so for analysis of the Avcoat heatshield material for the Orion Crew Module.]

\section{Multicomponent Ablation Equations}

A general theory for calculation of the thermochemical ablation rate of pyrolyzing TPS materials was presented in Reference 2. This theory was incorporated into the MAT code that included capabilities for simultaneous ablation, pyrolysis, surface element constraints, non-equilibrium surface reactions, and material failure. Several changes to the equations and to the solution methodology were required for dynamic integration with the SEB boundary condition equation. This section describes in detail the governing equations and solution methodology for multispecies thermochemical ablation as implemented in FIATC.

Figure 1 presents an illustration of the mass fluxes at the surface of a thermal protection material in a boundarylayer flow. A thin control volume is positioned just above the surface. The symbol $m$ is mass flux, and the mass fractions of elements in the charred solid, the pyrolysis gas, and the boundary-layer edge gas are denoted as $Y_{k c}$, $Y_{k g}$, and $Y_{k e}$, respectively. We allow one condensed species with mass fractions $Y_{k l_{f}}$ to fail.

For steady conditions there is no accumulation of elements in the control volume; therefore, element conservation is

\footnotetext{
$\$$ Our implementation differs significantly from that of Reference 6 in which MAT was used as a subroutine to generate partial $B^{\prime}$ tables at each time step within FIAT.
} 


$$
m_{g} Y_{k g}+m_{c} Y_{k c}=J_{k w}+(\rho V)_{w} Y_{k w}+m_{f} Y_{k l_{f}}
$$

which summed over all elements yields the mass balance

$$
m_{g}+m_{c}=(\rho V)_{w}+m_{f}
$$

because the sum of the diffusion fluxes $J_{k w}$ is zero.

Following theory presented in References 1 and 3, the diffusion flux is approximated using a transfer potential methodology, specifically

$$
j_{k w}=\rho_{e} u_{e} C_{M}\left(Z_{k w}-Z_{k e}\right)
$$

where $Z$ is a diffusion-coefficient-weighted average of mass and mole fractions. For equal diffusion coefficients, $Y$ and $Z$ are equal. With this assumption, elimination of $(\rho V)_{w}$ and $j_{k w}$ from Equation (1), division by $\rho_{e} u_{e} C_{M}$, and rearrangement of terms yields the element flux balance ${ }^{\S}$

$$
\left(1+B_{g}^{\prime}+B_{c}^{\prime}-B_{f}^{\prime}\right) Y_{k w}=Y_{k e}+B_{g}^{\prime} Y_{k g}+B_{c}^{\prime} Y_{k c}-B_{f}^{\prime} Y_{k l_{f}}
$$

where $B^{\prime}=m / \rho_{e} u_{e} C_{M}$ is a non-dimensional mass flux. The element flux balance insures that the correct elemental composition $Y_{k w}$ is obtained in the gas phase at the surface.

We consider next the equations for the case of equilibrium chemistry in the control volume of Figure 1 . We assume the system contains a mixture of $K$ elements, $I$ gaseous species, and $L$ condensed (liquid or solid) species. Simple sums over the gaseous species provide the mixture pressure

$$
P=\sum_{i} P_{i}
$$

the mixture enthalpy

$$
P \mathcal{M} h_{w}=\sum_{i} h_{i} P_{i}
$$

and the gaseous mass fraction at the wall

$$
P \mathcal{M} Y_{k w}=\mathcal{M}_{k} \sum_{i} c_{k i} P_{i}
$$

In these equations, the un-subscripted $\mathcal{M}$ is the mixture molecular weight.

Next, we select $K$ gaseous species (symbolically denoted as $N_{k}$ ) to represent base species for the elements in the system. Non-base gaseous species $N_{j}$ are represented by formation reactions of the base species

$$
\sum_{k} v_{k j} N_{k} \rightarrow N_{j}
$$

where $v_{k j}$ is obtained by inverting the relation $c_{k^{\prime} j}=c_{k^{\prime} k} v_{k j}$ (see nomenclature). ${ }^{* *}$ These formation reactions have temperature-dependent equilibrium constants

\footnotetext{
${ }^{\S}$ For unequal diffusion coefficients Equation (4) is more complex. Specifically, the right-hand side contains both $Z$ and $Y$ coefficients, and the left-hand side includes a sum over species pressures. Details, found in Reference 3 , were omitted here to save a page of text.

** Base species must have nonzero partial pressures and possess a nonsingular stoichiometry submatrix $c_{k^{\prime} k}$. There must be at least one gaseous species containing each element. Otherwise, the non-present element must be removed from the equation set.
} 


$$
\mathcal{K}_{j}(T)=P_{j} \prod_{k} P_{k}^{-v_{k j}}
$$

that are calculated from the species thermodynamic data as a function of temperature $T$.

Condensed species $N_{l}$ also are represented by formation reactions of the gaseous base species:

$$
\sum_{k} v_{k l} N_{k} \rightarrow N_{l}
$$

These formation reactions have temperature dependent equilibrium constants defined as

$$
\mathcal{K}_{l}(T)=X_{l} \prod_{k} P_{k}^{-v_{k l}}
$$

The coefficients $X_{l}$ account for the fact that $L$ condensed species share the available surface area. If the mixture of condensed species is ideal, then $X_{l}$ are surface mole fractions. With this interpretation, we add the constraint

$$
\sum_{l} X_{l}=1
$$

that allows condensed species to share the available surface area.

In the preceding equations, the known quantities are: $P, T, B_{g}^{\prime}, v_{k n}$, all element fractions $Y$, and thermodynamic data required for calculation of species enthalpies and equilibrium constants. The number of unknown variables and independent equations is summarized in Table 1. One additional equation is needed to determine the failure rate $B_{f}^{\prime}$. In the absence of material failure, we set $B_{f}^{\prime}=0$ to close the equation set. If a failure temperature is specified for a condensed species, different scenarios are possible. If the fail species is the only surface species, then when $T$ reaches $T_{\text {fail }}$ a range of $B_{f}^{\prime}$ is possible up to some maximum value that drives one $Y_{k w}$ to zero in Equation (4). Or if the failing species is one of multiple surface species, then a range of $B_{f}^{\prime}$ is possible for any $T \geq T_{\text {fail }}$. In either case, it is possible to iterate using prescribed values of $B_{f}^{\prime}$ until we find the maximum value that drives one $Y_{k w}$ to zero. The goal is to use the maximum failure rate when a range of failure rates is possible; otherwise, the solution is not unique. [This iteration is inefficient computationally. We are investigating what is the best way to implement material failure for carbon/glass systems.]

MAT includes an option to specify $M$ reversible or irreversible heterogeneous reactions in the form

$$
\sum_{n} \mu_{m i}^{R} N_{i} \rightarrow \sum_{n} \mu_{m i}^{P} N_{i}
$$

Table 1. Equations and unknowns

\begin{tabular}{|c|c|c|c|}
\hline Variables & $\begin{array}{c}\text { Number of } \\
\text { such variables }\end{array}$ & Equation Number & $\begin{array}{c}\text { Number of } \\
\text { such equations }\end{array}$ \\
\hline$P_{i}$ & $I$ & $(4)$ & $K$ \\
\hline$X_{l}$ & $L$ & $(5)$ & 1 \\
\hline$Y_{k w}$ & $K$ & $(6)$ & 1 \\
\hline $\mathcal{M}$ & 1 & $(7)$ & $I-K$ \\
\hline$h_{w}$ & 1 & $(9)$ & $L$ \\
\hline$B_{c}^{\prime}$ & 1 & $(10)$ & 1 \\
\hline$B_{f}^{\prime}$ & 1 & Total equations: & $I+K+L+3$ \\
\hline Total variables: & $I+K+L+4$ & &
\end{tabular}


Each reaction consumes the same condensed-phase species (typically carbon) denoted as $N_{l^{\prime}}$. The net forward reaction rate is expressed as

$$
\vec{R}_{m}=\vec{r}_{m}\left[X_{m l} \prod_{i} P_{i}^{\mu_{m i}^{R}}-\mathcal{K}_{m}^{-1} \prod_{i} P_{i}^{\mu_{m i}^{P}}\right]
$$

The forward rate is written in Arrhenius form

$$
\vec{r}_{m}=A_{m} T^{\phi_{m}} \exp \left(-E_{m} / T\right)
$$

For irreversible reactions, the second term on the right-hand side of Equation (11) is omitted. There may be one or two gaseous reactants and products in each reaction. For carbon ablation a variety of reactions are possible. In this work we employ a modern model from Park: ${ }^{6}$

$$
\begin{aligned}
& C_{(s)}+O \rightarrow C O \\
& C_{(s)}+N \rightarrow C N \\
& 3 C_{(s)} \rightarrow C_{3}
\end{aligned}
$$

We omit the pseudo-reaction $\mathrm{O}_{2}+\mathrm{C} \rightarrow 2 \mathrm{CO}$ that is found in some older models.

The chemical reactions are implemented as a conversion of one pseudo-element in the condensed species into another element of the same atomic weight in one of the product species. The quantity

$$
B_{k r}^{\prime}=\frac{\mathcal{M}_{k}}{\rho_{e} u_{e} C_{M}} \sum_{n} \sum_{m}\left(\mu_{m n}^{R}-\mu_{m n}^{P}\right) c_{k n} \vec{R}_{m}
$$

which is the dimensionless consumption rate of element $k$ by chemical reactions, appears as an extra term in the element flux balance.

$$
\left(1+B_{g}^{\prime}+B_{c}^{\prime}-B_{f}^{\prime}\right) Y_{k w}=Y_{k e}+B_{g}^{\prime} Y_{k g}+B_{c}^{\prime} Y_{k c}-B_{f}^{\prime} Y_{k l_{f}}-B_{k r}^{\prime}
$$

Examples of kinetic ablation of carbon phenolic will be presented in the Results section.

\section{Chemistry Solution Procedures}

The nonlinear equation set is solved by a Newton-Raphson procedure. There are advantages to using logs of some positive quantities as variables; therefore, the primary unknowns are chosen to be the vector $\mathbf{X}=\left[Y_{k w}, \ln P_{k}, B_{c}^{\prime}, \ln P \mathcal{M}, h_{w}, B_{f}^{\prime}, \ln P_{j}, \ln X_{l}\right]$. The Appendix contains a summary of the equation errors and partial derivatives for the Newton-Raphson solution. The error vector $\mathbf{E}$ can be any convenient ordering of Equations (A1-A7).

Given an initial guess for $\mathbf{X}$, the error vector $\mathbf{E}$ and the Jacobian matrix $\mathbf{J}=\partial \mathbf{E} / \partial \mathbf{X}$ may be assembled. The Newton-Raphson procedure is iteration of the following:

$$
\begin{aligned}
& \Delta \mathbf{X}=-\mathbf{J}^{-1} \mathbf{E} \\
& \mathbf{X}=\mathbf{X}+\Delta \mathbf{X}
\end{aligned}
$$

In practice, the corrections to $\mathbf{X}$ are damped slightly, and provided $\mathbf{J}$ remains nonsingular, iteration of Equations (15ab) drives the error vector to zero, thereby yielding the solution $\mathbf{X}$. Using the converged solution, partial derivatives with respect to any parameter may be determined as 


$$
\frac{\partial \mathbf{X}}{\partial(\text { parameter })}=\mathbf{J}^{-1} \frac{\partial \mathbf{E}}{\partial(\text { parameter })}
$$

This feature is very useful, because at almost negligible additional computational cost, the derivatives of $B_{c}^{\prime}$ and $h_{w}$ with respect to $B_{g}^{\prime}$ and $T$ may be calculated.

For many ablative materials the total number of possible gas-phase species is large, especially in hydrocarbon mixtures from pyrolyzing ablators. As the number of gas-phase species increases, it becomes inefficient to factorize or to invert the large Jacobian matrix. However, most of the governing equations are the equilibrium-constant relations for non-base species. Each of these equations references only one non-base species (either $P_{j}$ or $X_{l}$ ); therefore, the Jacobian matrix contains an identity submatrix that results from the terms $\partial \ln \mathcal{K}_{j} / \partial \ln P_{j^{\prime}}=\delta_{j j^{\prime}}$ and $\partial \ln \mathcal{K}_{l} / \partial \ln P_{l^{\prime}}=\delta_{l l^{\prime}}$ in Equations (A6-A7). Therefore, these equations may be used to eliminate corrections $\ln P_{j}$ and $\ln X_{l}$ that appear in the other equations.

To implement this submatrix reduction, we define primary and secondary unknowns as listed in Table 2 . The equations are ordered such that the equilibrium expressions are the final $I+L-K$ equations. Equation (15a) is rewritten as

$$
\mathbf{J} \Delta \mathbf{X}=\left[\begin{array}{ll}
\mathbf{A} & \mathbf{B} \\
\mathbf{C} & \mathbf{I}
\end{array}\right]\left[\begin{array}{c}
\Delta \mathbf{X}_{1} \\
\Delta \mathbf{X}_{2}
\end{array}\right]=-\left[\begin{array}{l}
\mathbf{E}_{1} \\
\mathbf{E}_{2}
\end{array}\right]
$$

where $\mathbf{A}$ is a square matrix of dimension $2 K+4$, and $\mathbf{I}$ is an identity matrix of size $I+L-K$. Submatrix reduction is the elimination of $\mathbf{B}$ to produce

$$
\left[\begin{array}{ll}
\mathbf{A}^{\prime} & 0 \\
\mathbf{C} & \mathbf{I}
\end{array}\right]\left[\begin{array}{l}
\Delta \mathbf{X}_{1} \\
\Delta \mathbf{X}_{2}
\end{array}\right]=-\left[\begin{array}{l}
\mathbf{E}_{1}^{\prime} \\
\mathbf{E}_{2}
\end{array}\right]
$$

where $\mathbf{A}^{\prime}=\mathbf{A}-\mathbf{B C}$ and $\mathbf{E}_{1}^{\prime}=\mathbf{E}_{1}-\mathbf{B} \mathbf{E}_{2}$. The solution is then

$$
\begin{gathered}
\Delta \mathbf{X}_{1}=-\left[\mathbf{A}^{\prime}\right]^{-1} \mathbf{E}_{1}^{\prime} \\
\Delta \mathbf{X}_{2}=-\mathbf{E}_{2}-\mathbf{C} \Delta \mathbf{X}_{1}
\end{gathered}
$$

The advantage of the submatrix reduction is the large computational savings from inverting or factorizing the small matrix $\mathbf{A}^{\prime}$ rather than the large matrix $\mathbf{J}$, which more than offsets the expense of the simple algebra involved in Equations 17-19. Partial derivatives of solution variables (such as $\partial B_{c}^{\prime} / \partial T$ ) may still be calculated, provided the vector of partial derivatives is carried through the algebraic operations described above.

Convergence of the Newton-Raphson procedure will be adversely affected if, by poor choice of base species, some $P_{k}$ is very small compared with $P_{j}$ for other species that contain element $k$. The solution of this potential problem is to dynamically reselect base species as required during the course of the solution. The approach used in FIATC is to select the species with the largest pressure containing each element. The matrix $v_{k n}$ depends on the choice of base species, but all other matrix operations are automated by use of pointer arrays for base and nonbase species.

Trace species partial pressures or surface mole fractions may become too small to compute accurately. Provisions are made in FIATC to disregard species with mole fractions below a prescribed cutoff value (of $10^{-99}$ ). Non-base species at or below the cutoff value and with negative corrections to $\ln P_{j}$ or $\ln X_{l}$ are removed from the equations and not considered in numerical convergence tests.

Table 2. Variable sequencing for submatrix reduction

\begin{tabular}{|l|l|l|l|}
\hline Vector & Designation & Variables & Length \\
\hline $\mathbf{X}_{1}$ & primary & $Y_{k w}, \ln P_{k}, \ln B_{c}^{\prime}, \ln P \mathcal{M}, h_{w}, B_{f}^{\prime}$ & $2 K+4$ \\
\hline $\mathbf{X}_{2}$ & secondary & $\ln P_{j}, \ln X_{l}$ & $I+L-K$ \\
\hline
\end{tabular}




\section{Surface Energy Balance}

We begin with a general form of the surface energy balance for unequal diffusion coefficients, that is slightly modified from that presented in Reference 3 (equation 78, page 48)

$$
\begin{aligned}
\rho_{e} u_{e} C_{H}\left(H_{r}-h_{e w}\right)+\rho_{e} u_{e} C_{M}\{ & \left.\sum\left(Z_{i e}-Z_{i w}\right) h_{i}^{T_{w}}-\left(B_{c}^{\prime}+B_{g}^{\prime}-B_{f}^{\prime}\right) h_{w}\right\} \\
& +m_{c} h_{c}+m_{g} h_{g}-m_{f} h_{l_{f}}=F \sigma \varepsilon_{w}\left(T_{w}^{4}-T_{\infty}^{4}\right)+q_{\mathrm{cond}}-\alpha_{w} q_{\mathrm{rad}}
\end{aligned}
$$

Here $h_{e w}$ is the enthalpy of the edge gas (frozen composition) evaluated at the wall temperature. The first term on the left-hand side is sensible convective heat flux; that is, the convective heat flux that would occur for a frozen boundary layer and a non-catalytic wall. The rest of the left-hand side includes all contributions from chemical reactions. The right-hand side terms are self-explanatory.

In traditional CMA methodology, edge-gas tables provide $h_{e w}$ and $\sum Z_{i e} h_{i}^{T_{w}}$ as functions of $T$ and $P$, and thermochemical-ablation tables provide $B_{f}^{\prime}, h_{w}, T$, and $\sum Z_{i w} h_{i}^{T_{w}}$ as functions of the independent variables $P, B_{g}^{\prime}$, and $B_{c}^{\prime}$. Both sets of tables apply to a specific edge gas composition. If the edge-gas composition changes as a function of time, the only feasible approach is to pick a fixed edge composition to use for the entire heating environment under consideration. Clearly this approach has questionable accuracy for trajectory-based environments. [Varying edge-gas composition will be feasible with FIATC after we add the capability for unequal diffusion coefficients. The quantity $h_{e w}$ can be estimated using the chemistry subroutines provided the edge composition is specified as a function of time.] CMA allows input of a single value for $C_{M} / C_{H}$. For unequal diffusion coefficients, Reference 3 recommends $C_{M}=C_{H}(L e)^{2 / 3}$ where Le is the Lewis number. For equal diffusion coefficients $\sum Z_{i e} h_{i}^{T_{w}}=h_{e w}$ and $\sum Z_{i w} h_{i}^{T_{w}}=h_{w}$; however, the quantity $h_{e w}$ still is required.

For equal diffusion coefficients and $C_{M}=C_{H}$, Equation (20) reduces to a more recognizable expression

$$
\rho_{e} u_{e} C_{H}\left\{\left(H_{r}-h_{w}\right)+B_{c}^{\prime}\left(h_{c}-h_{w}\right)-B_{f}^{\prime}\left(h_{l_{f}}-h_{w}\right)\right\}+m_{g}\left(h_{g}-h_{w}\right)=F \sigma \varepsilon_{w}\left(T_{w}^{4}-T_{\infty}^{4}\right)+q_{c o n d}-\alpha_{w} q_{r a d}
$$

For consistency with the coupling methodology described in the next section, the char and fail terms were moved inside the braces, whereas the pyrolysis gas terms were moved outside the braces.

A blowing correction accounts for the reduction in heat transfer coefficient due to the injection of pyrolysis and ablation-product gases into the boundary layer. The blowing correction equation used by FIATC is

$$
\frac{C_{H}}{C_{H 1}}=\frac{\ln \left(1+2 \lambda B^{\prime}\right)}{2 \lambda B^{\prime}} \quad \text { where } \quad B^{\prime}=\frac{\left(m_{c}+m_{g}-m_{f}\right)}{\rho_{e} u_{e} C_{M}}
$$

Here $\lambda$ is the blowing reduction parameter, and $C_{H} / C_{H 1}$ is the ratio of the blown (ablating) to the unblown (nonablating) heat transfer coefficients. The fail mass flux does not contribute to the blowing correction. For laminar flow $\lambda=0.5$ or higher depending on the geometry and the ratio of molecular weights of the injected and boundarylayer-edge gas. For transitional or turbulent flow smaller values of $\lambda$ are used.

\section{Coupling Implementation}

In FIATC the thermal-response solver calculates $m_{g}, T, q_{\text {cond }}$ and density at all points within the solid including a surface node. The SEB from Equation (21) is a boundary condition. At each time step, the quantities $H_{r}, P, q_{\mathrm{rad}}$, and $\rho_{e} u_{e} C_{H 1}$ are interpolated from environment tables. Then, the following steps are iterated. Based on current values, thermal properties for internal points and surface properties such as $\alpha_{w}, \varepsilon_{w}, h_{c}, h_{g}$, and $h_{l_{f}}$ are

\footnotetext{
${ }^{\dagger}$ In this formulation $B_{f}^{\prime}$ is a portion of $B_{c}^{\prime}$; that is, $B_{c}^{\prime}$ represents the total solid ablation rate. However, in some codes $B_{f}^{\prime}$ is treated as an independent quantity that is not included in $B_{c}^{\prime}$. It is important to determine which definition is used in any ablation tables with non-zero fail.
} 
calculated. Using the current value of $\rho_{e} u_{e} C_{H}$, the surface value of $m_{g}$ is converted into $B_{g}^{\prime}$. The values of $B_{g}^{\prime}$, P, and $T_{w}$ are input to the chemistry module that calculates $B_{c}^{\prime}, B_{f}^{\prime}$, and $h_{w}$ as well as derivatives with respect to $B_{g}^{\prime}$ and $T_{w}$. These quantities are loaded into the SEB equation of the thermal solver. The thermal solver uses a NewtonRaphson solution method, and the partial derivatives from the chemistry solution assist in convergence of the SEB equation. Typically 3 to 5 iterations are required to converge all equations including the SEB.

For nonablating materials, the surface mass fluxes are zero, and the SEB simplifies to

$$
\rho_{e} u_{e} C_{H 1}\left(H_{r}-h_{w}\right)=F \sigma \varepsilon_{w}\left(T_{w}^{4}-T_{\infty}^{4}\right)+q_{c o n d}-\alpha_{w} q_{r a d}
$$

In this case, the chemistry module calculates $h_{w}$ and $\partial h_{w} / \partial T_{w}$.

Species and elemental information are required to implement the dynamic surface chemistry option in FIATC. This information is placed in the environment and material property input files. At the beginning of the environment data file, the user inputs one of the following nine keywords: Venus, Earth, Mars, Jupiter, Saturn, Uranus, Neptune, Titan, or Elements. If one of the first eight keywords is input, FIATC uses the standard atmosphere for the selected planet or moon (Table 2). For Earth the table lists the composition of dry air, ${ }^{\sharp}$ and for Venus we omitted the trace amount of $\mathrm{SO}_{2}$. If the keyword Elements is used, then FIATC reads the number of elements, their atomic weights, and their mass fractions in the edge gas. We provide a default database that contains JANNAF thermodynamic data of 91 gaseous species containing the elements $\mathrm{H}, \mathrm{He}, \mathrm{N}, \mathrm{O}, \mathrm{Si}$, and/or Ar. This database is adequate for most flight or test atmospheres and for many thermal protection materials.

In the material property file, each material has a type designation. Specifically, type 1 is reusable, type 3 is ablating but non-pyrolyzing, and type 4 is ablating and pyrolyzing. New choices in FIATC are types 5 and 6 , which are the same as types 3 and 4, respectively, but without $B^{\prime}$ tables. For materials of types 5 and 6 , new inputs are the number of elements, their atomic weights, and their mass fractions in the char and in the pyrolysis gas, followed by JANNAF data for the condensed-phase species under consideration, and finally data for up to five heterogeneous surface reactions. Calculations with dynamic surface chemistry may be performed with any materials of type 1, 5, or 6. For surface materials of type 1, the simplified SEB from Equation (23) is used. [A material of type 2, pyrolyzing but non-ablating, also is feasible; however, we have not yet implemented this option with the dynamic surface chemistry. There has been little interest in this type of material.]

Table 3. Known atmospheres

\begin{tabular}{|c|c|c|c|c|c|c|c|c|}
\hline \multirow{2}{*}{ Species } & \multicolumn{8}{|c|}{ Mole Fractions (from National Space Science Data Center fact sheets, at http://nssdc.gsfc.nasa.gov) } \\
\hline & Venus & Earth & Mars & Jupiter & Saturn & Uranus & Neptune & Titan \\
\hline $\mathrm{H}_{2}$ & & & & 0.898 & 0.963 & 0.825 & 0.80 & \\
\hline $\mathrm{He}$ & & & & 0.102 & 0.0325 & 0.152 & 0.19 & \\
\hline $\mathrm{CO}_{2}$ & 0.965 & 0.00038 & 0.9532 & & & & & \\
\hline $\mathrm{CH}_{4}$ & & & & 0.003 & 0.0045 & 0.023 & 0.015 & 0.016 \\
\hline $\mathrm{N}_{2}$ & 0.035 & 0.78084 & 0.027 & & & & & 0.984 \\
\hline $\mathrm{O}_{2}$ & & 0.20946 & 0.0013 & & & & & \\
\hline $\mathrm{Ar}$ & & 0.00934 & 0.016 & & & & & \\
\hline $\mathrm{H}_{2} \mathrm{O}$ & & & 0.00021 & & & & & \\
\hline $\mathrm{CO}$ & & & 0.0008 & & & & & \\
\hline $\mathrm{NH}_{3}$ & & & & 0.00026 & 0.000125 & & & \\
\hline NO & & & 0.0001 & & & & & \\
\hline \multirow{2}{*}{ Element } & \multicolumn{8}{|c|}{ Mass Fractions (normalized from the species tabulated above) } \\
\hline & Venus & Earth & Mars & Jupiter & Saturn & Uranus & Neptune & Titan \\
\hline Hydrogen & & & 0.0000098 & 0.8027572 & 0.9133652 & 0.6649572 & 0.6401139 & 0.0023185 \\
\hline Helium & & & & 0.1797725 & 0.0606279 & 0.2304166 & 0.2909562 & \\
\hline Carbon & 0.2667604 & 0.0001576 & 0.2639229 & 0.0158667 & 0.0251909 & 0.1046262 & 0.0689299 & 0.0069075 \\
\hline Nitrogen & 0.0225654 & 0.7551531 & 0.0174533 & 0.0016036 & 0.0008160 & & & 0.9907740 \\
\hline Oxygen & 0.7106742 & 0.2318084 & 0.7038923 & & & & & \\
\hline Argon & & 0.0128809 & 0.0147217 & & & & & \\
\hline
\end{tabular}

\# For Earth the water vapor fraction is significant but highly variable in the troposphere. In the stratosphere (static pressure below about $10 \mathrm{kPa}$ ) the water fraction generally is below that of $\mathrm{CO}_{2}$. 


\section{Results}

[For the final paper we are considering a comparison of equilibrium and finite-rate PICA ablation for the following problems: 1) an arcjet environment, 2) thickness sizing for an Orion environment, 3) Stardust, and 4) an MSL environment. Avcoat calculations probably will be reported in a separate paper.]

For this extended abstract, we consider two examples of ablation of phenolic impregnated carbon ablator (PICA). In previous work, an equilibrium ablation and thermal property model for PICA was developed. ${ }^{8}$ The model was validated by comparison of predictions with arcjet data over a wide range of stagnation test conditions. ${ }^{9}$ The predictions compared well with the data for surface temperature and recession for stagnation point heat flux above $100 \mathrm{~W} / \mathrm{cm}^{2}$. However, for heat fluxes below $80 \mathrm{~W} / \mathrm{cm}^{2}$, corresponding to surface temperatures below $2000 \mathrm{~K}$, the equilibrium model overpredicts the ablation rate, and a kinetic rate model for carbon ablation is recommended. ${ }^{10}$

In Reference 10, the lowest test condition was an air/argon mixture with measured stagnation heat flux and pressure of $40 \mathrm{~W} / \mathrm{cm} 2$ and $4.4 \mathrm{kPa}$, respectively. The exposure duration was 200 seconds. The PICA model and facility calorimeter both had the iso- $q$ shape, shown in Figure 2, with $10.16 \mathrm{~cm}$ diameter. This shape is used in testing in the NASA Ames arcjets, because the surface heating is relatively constant over the front face of the model. Following procedures described in Reference 9, facility data and flowfield analyses were combined to obtain boundary conditions for the material-response analysis. Specifically, in this case the centerline enthalpy and unblown heat transfer coefficient were estimated as $5.2 \mathrm{MJ} / \mathrm{kg}$ and $0.077 \mathrm{~kg} / \mathrm{m}^{2}$-s, respectively.

Reference 9 describes in detail some of the experimental uncertainties in arcjet testing. In order to assess the effect of these uncertainties on model predictions, our current procedure is to perform arcjet simulations using the nominal heating conditions and also using scaling factors of $\pm 10 \%$ applied to the heat transfer coefficient. This procedure was followed using FIATC with the equilibrium ablation model for PICA, and the results are presented in Figure 3. The yellow-shaded region is the range of predicted recession. The black dots are the measured centerline recession for the two tested samples with error bars of $\pm 0.5 \mathrm{~mm}$. The equilibrium model overpredicts the measured recession by approximately $45 \%$.

The FIATC calculations were repeated using the reaction model for ablation of carbon phenolic in Equation (13) with rate parameters from Reference 7. The kinetic model predicts lower recession than the equilibrium model, and the range of predictions overlaps the data as shown in Figure 4. The predicted surface blowing rates are plotted in Figure 5. In this environment, the carbon ablation rate is predominantly a result of the reaction $C_{(s)}+O \rightarrow C O$. The surface does not reach a steady state of ablation, although conditions are relatively constant over the last 50 seconds of heating. The char flux increases continuously, whereas the pyrolysis gas flux peaks within the first second of exposure. The total blowing rate peaks near 25 seconds, then decreases $5 \%$ over the next 175 seconds.

The unblown heat transfer coefficient is considered to be constant for the entire test duration; however, the blown coefficient depends on the total surface mass flux according to the blowing correction in Equation (22). The maximum $B_{g}^{\prime}+B_{c}^{\prime}$ is 0.174 , which is results in a minimum blowing correction of 0.922 ; therefore, the minimum blown coefficient is $0.071 \mathrm{~kg} / \mathrm{m}^{2}$-s (Figure 6). Also, except for the first 10 seconds of exposure, the blown coefficient is relatively constant. Therefore, for this specific arcjet case, approximate predictions could be obtained using kinetic ablation tables generated for a fixed blown coefficient of about $0.0713 \mathrm{~kg} / \mathrm{m}^{2}-\mathrm{s}$. However, in general, this approach is not accurate; instead, FIATC should be used to dynamically calculate the ablation rate without resorting to such assumptions.

We compared FIATC predictions for kinetic and equilibrium ablation models with measured recession of PICA in arcjet tests over a broad range of stagnation conditions. The results are summarized in Figure 7. The equilibrium ablation model accurately predicts the recession for surface temperatures above $2000 \mathrm{~K}$ (or heat flux above 80 $\mathrm{W} / \mathrm{cm}^{2}$ ). For surface temperatures below $2000 \mathrm{~K}$, rate limitations to carbon oxidation become important, and the kinetic ablation model is required. Below about $1000 \mathrm{~K}$ the recession rate is negligible.

Additionally, for pressure below about $7 \mathrm{kPa}$, the pyrolysis gas should be modeled as inert when it reaches the ablating surface. Pyrolysis gas is a multi-component hydrocarbon mixture that reacts with oxygen in the flowfield by a number of two- and three-body reactions. These homogeneous reactions have rates that are at least second in pressure. At sufficiently low pressure, these reactions become slower than the heterogeneous reaction for oxidation of solid carbon that is first-order in pressure, and it is incorrect to equilibrate the pyrolysis gas with the oxygen in the atmospheric gas.

In our work to date, the kinetic model approaches, but does not reach, full equilibrium at high temperatures. Consequently, the kinetic model underpredicts the measured recession for conditions above $200 \mathrm{~W} / \mathrm{cm}^{2}$. We are continuing our research in this area, because we would like to have a single model that may be used for the entire 
range of flight conditions and which, of course, agrees with the entire set of stagnation test data. Our current procedure is to begin calculations using the kinetic model, then to switch to the equilibrium model for surface temperature above $2250 \mathrm{~K}$. This hybrid model is inelegant, but consistent with the arcjet test data.

The second example is a flight environment from a recent design cycle for the Orion Crew Module. Figure 8 presents the margined heat fluxes at a critical location on the windward shoulder. The pressure history is shown in Figure 9. The vehicle first performs a skip through the upper atmosphere, then re-enters the atmosphere for a direct entry. Consequently there are two heat pulses. The first pulse, corresponding to the skip maneuver, has shorter duration but higher pressure and heat flux. The second pulse has longer duration but lower heat flux with negligible radiation.

The total trajectory time is 1085 seconds; however, the ablative boundary conditions are turned off at 693 seconds as the velocity drops below Mach 1 . After this time we apply a radiative cooling boundary condition that allows pyrolysis to continue during the cooldown portion of the trajectory. The TPS stackup is PICA bonded to a titanium-based structure using RTV-560 adhesive. As part of the analysis, FIATC calculates the minimum PICA thickness consistent with a maximum PICA-to-RTV temperature of $561 \mathrm{~K}$. This temperature is the maximum reuse temperature of the adhesive. Orion flow environments are assumed to be fully turbulent; consequently, a blowingreduction parameter $\lambda=0.4$ was used in the material response analyses

FIATC calculations were performed using the equilibrium ablation model and the hybrid kinetic-equilibrium model. The predicted surface temperature (Figure 10) clearly shows the two heat pulses. For the hybrid ablation model, the equilibrium portion is required only during the hottest portion of the first heat pulse (the yellow-shaded region from 45 to 145 seconds), and the kinetic portion is used for the rest of the trajectory. The surface temperature is insensitive to the choice of ablation model, except at the tail end of the second heat pulse. After the second maximum in heat flux, the edge enthalpy decreases rapidly as the vehicle's velocity drops below Mach 1 , and the atomic oxygen fraction also decreases. The kinetic model therefore predicts a low ablation rate, whereas the equilibrium model predicts steady recession at the diffusion-limited oxidation plateau $\left(B^{\prime} c=0.167\right)$. The wall enthalpy is lower for the latter case (owing to the presence of $\mathrm{CO}$ ), and therefore the convective heating and surface temperature slightly larger. In both calculations the small kink at 693 seconds is a result of the switch in boundary conditions.

The predicted ablation rate is shown in Figures 11. The kinetic model predicts lower recession rate in the cool period between the two heat pulses, and in the cooldown portion of the second heat pulse, as described previously. The $60 \%$ difference in predicted total recession (Figure 12) is remarkable. The equilibrium model predicts non-zero recession in the time interval between heat pulses; however, this inaccuracy is minor compared with its gross overprediction of the total recession in the second heat pulse.

Interestingly, despite this large difference in predicted total recession, the calculated sizing to achieve the specified bond-line temperature was almost identical for the two cases: $5.71 \mathrm{~cm}$ for the equilbrium model and 5.76 $\mathrm{cm}$ for the hybrid model.

The surface blowing rates are presented in Figure 13. The char flux exceeds the pyrolysis gas flux during the highest heating portion of both heat pulses. The pyrolysis gas flux reaches a maximum in the first 50 seconds, then maintains a small value until beyond the end of the second heat pulse. There is no large increase in pyrolysis gas at the start of the second heat pulse, because any phenolic near the surface has already been fully charred.

[Finally, Figure 14 shows the blowing rates of major species as a function of time...]

[We will add a discussion of computational time for these calculations.]

\section{Concluding Remarks}

[The final paper will include a conclusions section.]

\section{References}

${ }^{1}$ Kendall, R.M., "An Analysis of the Coupled Chemically Reacting Boundary Layer and Charring Ablator, Part V, A General Approach to the Thermochemical Solution of Mixed Equilibrium-Nonequilibrium Homogeneous or Heterogeneous Systems," NASA CR-1064, June 1968.

${ }^{2}$ Milos, F.S. and Chen, Y.-K., "Comprehensive Model for Multicomponent Ablation Thermochemistry, " AIAA Paper $97-$ 0141, Jan. 1997.

${ }^{3}$ Moyer, C.B., and Rindal, R.A., "An Analysis of the Coupled Chemically Reacting Boundary Layer and Charring Ablator, Part II, Finite Difference Solution for the In-Depth Response of Charring Materials Considering Surface Chemical and Energy Balances," NASA CR-1061, June 1968.

${ }^{4}$ Chen, Y.-K., and Milos, F.S., "Ablation and Thermal Analysis Program for Spacecraft Heatshield Analysis, " Journal of Spacecraft and Rockets, Vol. 36, No. 3, 1999, pp. 475-483. 
${ }^{5}$ Milos, F.S., and Chen, Y.-K., "Ablation and Thermal Response Property Model Validation for Phenolic Impregnated Carbon Ablator," AIAA Paper 2009-0262, Jan. 2009.

${ }^{6}$ Beerman, A.F., Lewis, M.J., Starkey, R.P., and Cybyk, B.Z., "Significance of Nonequilibrium Surface Interactions in Stardust Return Capsule Ablation Modeling," Journal of Thermophysics and Heat Transfer, Vol. 23, No. 3, 2009 , pp. $425-431$.

${ }^{7}$ Park, C., "Calculation of Stagnation-Point Heating Rates Associated with Stardust Vehicle," Journal of Spacecraft and Rockets, Vol. 44, No. 1, 2007, pp. 24-32.

${ }^{8}$ Milos, F.S., and Chen, Y.-K., "Ablation and Thermal Property Model for Phenolic Impregnated Carbon Ablator (PICA)," NASA-TM-2009-215377, March 2009.

${ }^{9}$ Milos, F.S. and Chen, Y.-K., "Ablation and Thermal Response Property Model Validation for Phenolic Impregnated Carbon Ablator," AIAA Paper 2009-0262, Jan. 2009.

${ }^{10}$ Milos, F.S. and Chen, Y.-K., "Non-Equilibrium Ablation of Phenolic Impregnated Carbon Ablator," AIAA Paper 20100981, Jan. 2010.

other possible references

Balter-Peterson, A., Nichols, F., Mifsud, B., and Love, W., "Arc Jet Testing in NASA Ames Research Center Thermophysics Facilities, " AIAA Paper 1992-5041, Dec. 1992.

Greathouse, J.S., Kirk, B.S., Lillard, R.P., Truong, T.H., Robinson, P., Cerimele, C.J., "Crew Exploration Vehicle (CEV) Crew Module Shape Selection Analysis and CEV Aerosciences Project Overview," AIAA Paper 2007-0603, Jan. 2007.

\section{Appendix: Error Equations and Derivatives for Ablation Chemistry}

The following is a summary of the error equations and partial derivatives used by the chemistry module within FIATC.

\section{Element flux balance}

$$
\begin{aligned}
& E_{k}=\left(1+B_{g}^{\prime}+B_{c}^{\prime}-B_{f}^{\prime}\right) Y_{k w}-Y_{k e}-B_{g}^{\prime} Y_{k g}-B_{c}^{\prime} Y_{k c}+B_{f}^{\prime} Y_{k l_{f}}-B_{k r}^{\prime} \\
& \partial E_{k} / \partial Y_{k^{\prime} w}=\delta_{k k^{\prime}}\left(1+B_{g}^{\prime}+B_{c}^{\prime}-B_{f}^{\prime}\right) \\
& \partial E_{k} / \partial B_{c}^{\prime}=Y_{k w}-Y_{k c} \\
& \partial E_{k} / \partial B_{f}^{\prime}=Y_{k l_{f}}-Y_{k w} \\
& \partial E_{k} / \partial \ln P_{i}=-\partial B_{k r}^{\prime} / \partial \ln P_{i} \\
& \partial E_{k} / \partial \ln X_{l}=-\partial B_{k r}^{\prime} / \partial \ln X_{l} \\
& \partial E_{k} / \partial \ln T=-\partial B_{k r}^{\prime} / \partial \ln T \\
& \partial E_{k} / \partial B_{g}^{\prime}=Y_{k w}-Y_{k g}
\end{aligned}
$$

\section{Wall mass fraction sum}

$$
\begin{aligned}
& E_{k}=\mathcal{M}_{k} \sum_{i} c_{k i} P_{i}-P \mathcal{M} Y_{k w} \\
& \partial E_{k} / \partial Y_{k^{\prime} w}=-\delta_{k k^{\prime}} P \mathcal{M} \\
& \partial E_{k} / \partial \ln P_{i}=\mathcal{M}_{k} c_{k i} P_{i} \\
& \partial E_{k} / \partial \ln P \mathcal{M}=-P \mathcal{M} Y_{k w}
\end{aligned}
$$

\section{Pressure sum}

$$
\begin{aligned}
& E=\sum_{i} P_{i}-P \\
& \partial E / \partial \ln P_{i}=P_{i}
\end{aligned}
$$




\section{Mixture enthalpy sum}

$$
\begin{aligned}
& E=\sum_{i} h_{i} P_{i}-P \mathcal{M} h_{w} \\
& \partial E / \partial \ln P_{i}=h_{i} P_{i} \\
& \partial E / \partial \ln P \mathcal{M}=-P \mathcal{M} h_{w} \\
& \partial E / \partial h_{w}=-P \mathcal{M} \\
& \partial E / \partial \ln T=\sum_{i} C p_{i} T P_{i}
\end{aligned}
$$

\section{Surface fraction sum}

$$
\begin{aligned}
& E=\sum_{l} X_{l}-1 \\
& \partial E / \partial \ln X_{l}=X_{l}
\end{aligned}
$$

Material failure rate [currently]

$$
\begin{aligned}
& E=B_{f}^{\prime}-\left(B_{f}^{\prime}\right)_{\text {prescribed }} \\
& \partial E_{j} / \partial B_{f}^{\prime}=1
\end{aligned}
$$

\section{Equilibrium for non-base gaseous species}

$$
\begin{aligned}
& E_{j}=\ln P_{j}-\sum_{k} v_{k j} \ln P_{k}-\ln \mathcal{K}_{j} \\
& \partial E_{j} / \partial \ln P_{k}=-v_{k j} \\
& \partial E_{j} / \partial \ln P_{j^{\prime}}=\delta_{j j^{\prime}} \\
& \partial E_{j} / \partial \ln T=\left(\sum_{k} v_{k j} h_{k}-h_{j}\right) / R T
\end{aligned}
$$

\section{Equilibrium for condensed species}

$$
\begin{aligned}
& E_{l}=\ln X_{l}-\sum_{k} v_{k l} \ln P_{k}-\ln \mathcal{K}_{l} \\
& \partial E_{l} / \partial \ln P_{k}=-v_{k l} \\
& \partial E_{l} / \partial \ln X_{l^{\prime}}=\delta_{l l^{\prime}} \\
& \partial E_{l} / \partial \ln T=\underset{k}{\left(\sum_{k l} h_{k}-h_{l}\right) / R T}
\end{aligned}
$$




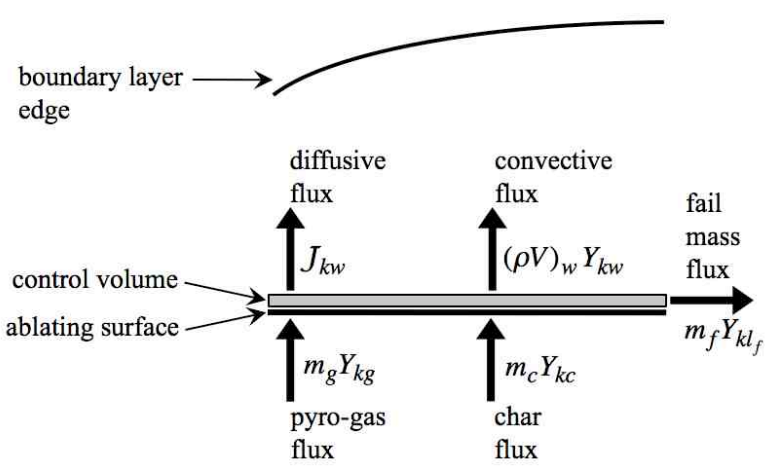

Figure 1. Mass fractions and compositions at the surface of a thermal protection material in a boundary layer.

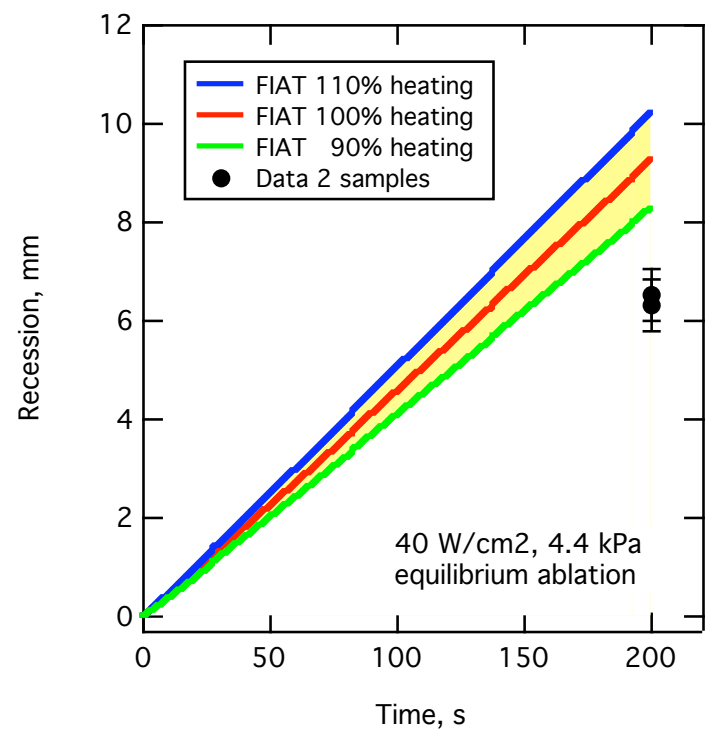

Figure 3. Measured centerline recession and predictions of equilibrium ablation model.

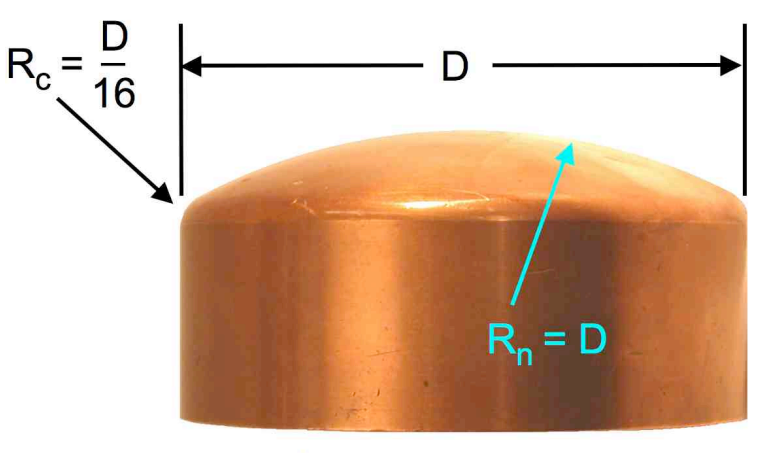

"Iso-q" Calorimeter

Figure 2. Model shape for stagnation arcjet tests. TPS samples have the same external shape as the calorimeter used to measure the heat flux and stagnation pressure.

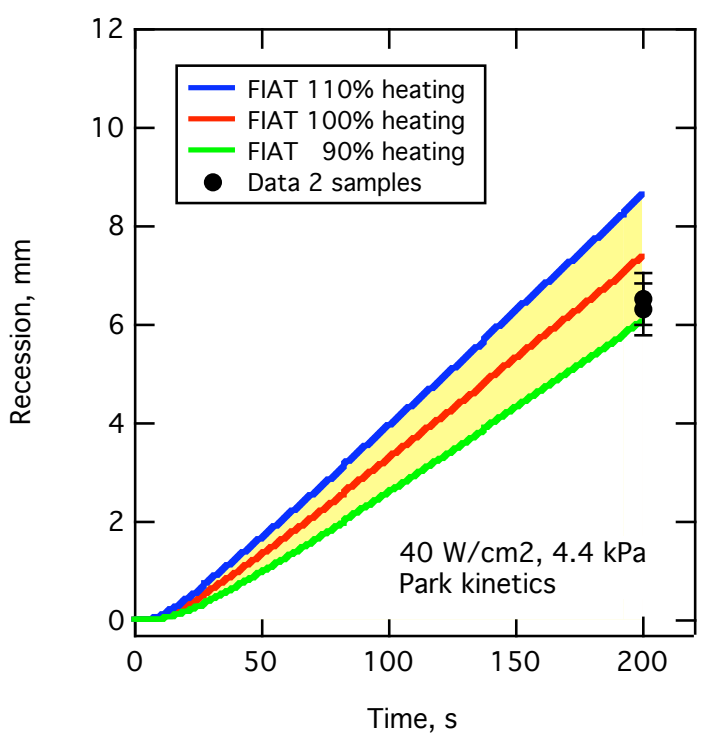

Figure 4. Measured centerline recession and predictions of kinetic ablation model. 


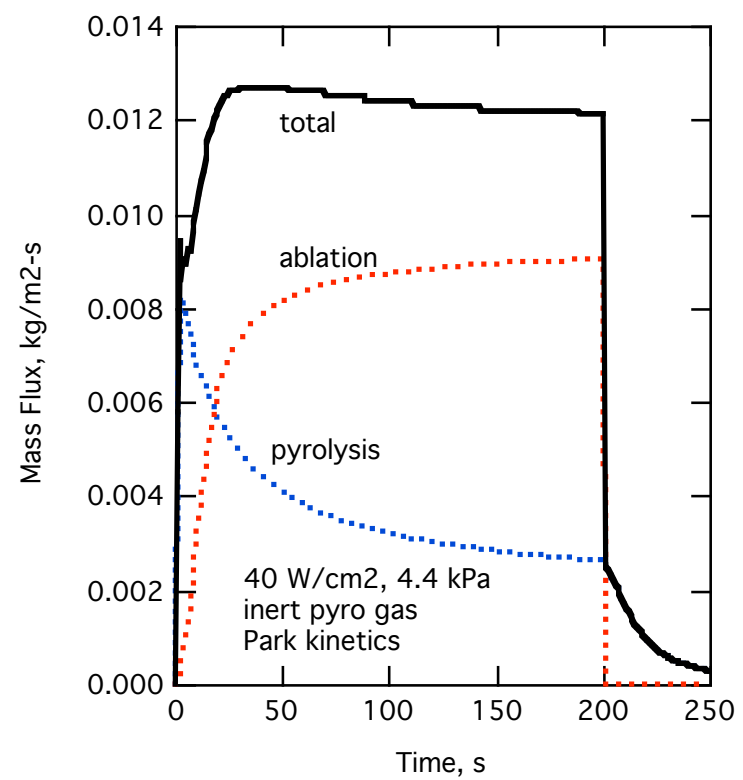

Figure 5. Predicted surface blowing rates for nominal arcjet environment.

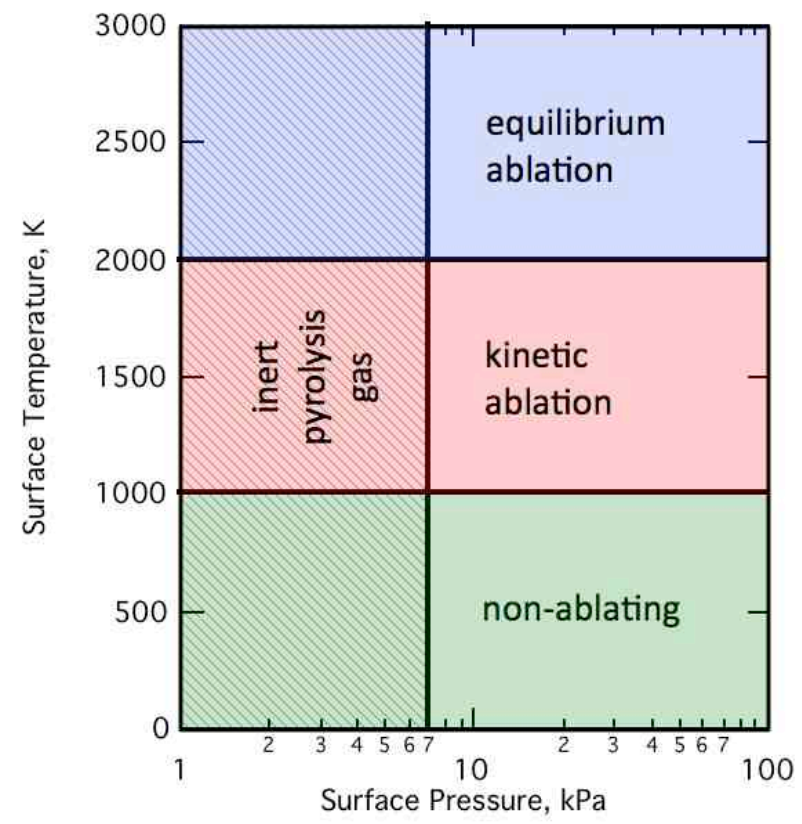

Figure 7. Nominal ablation regimes for PICA.

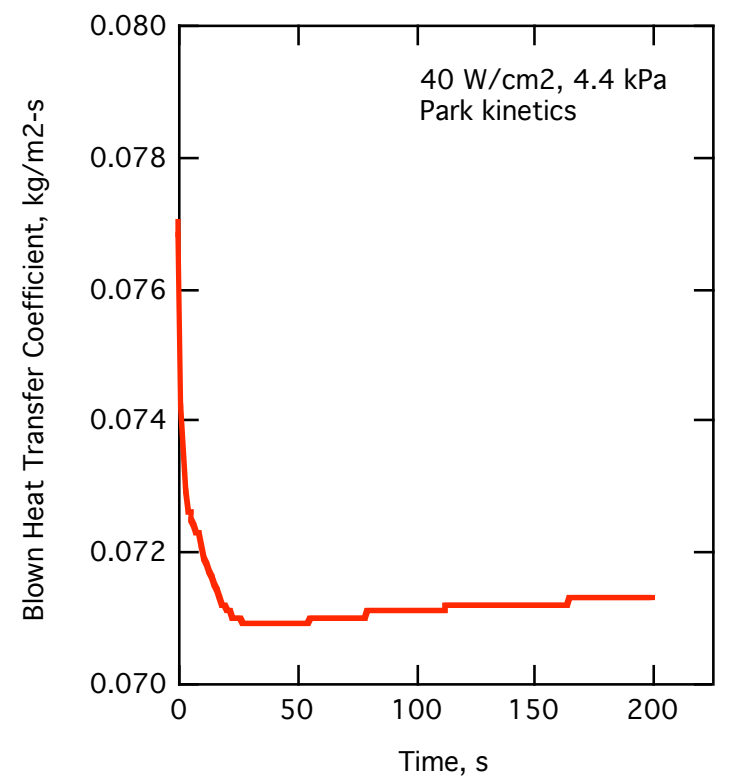

Figure 6. Predicted blown heat transfer coefficient for arcjet environment.

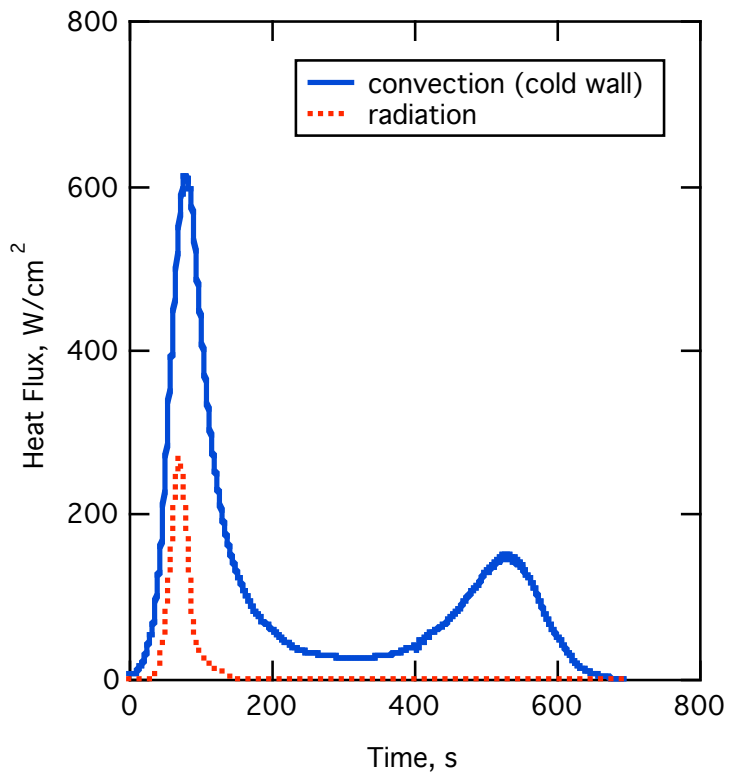

Figure 8. Margined heat fluxes at a critical shoulder location on the Orion Crew Module. 


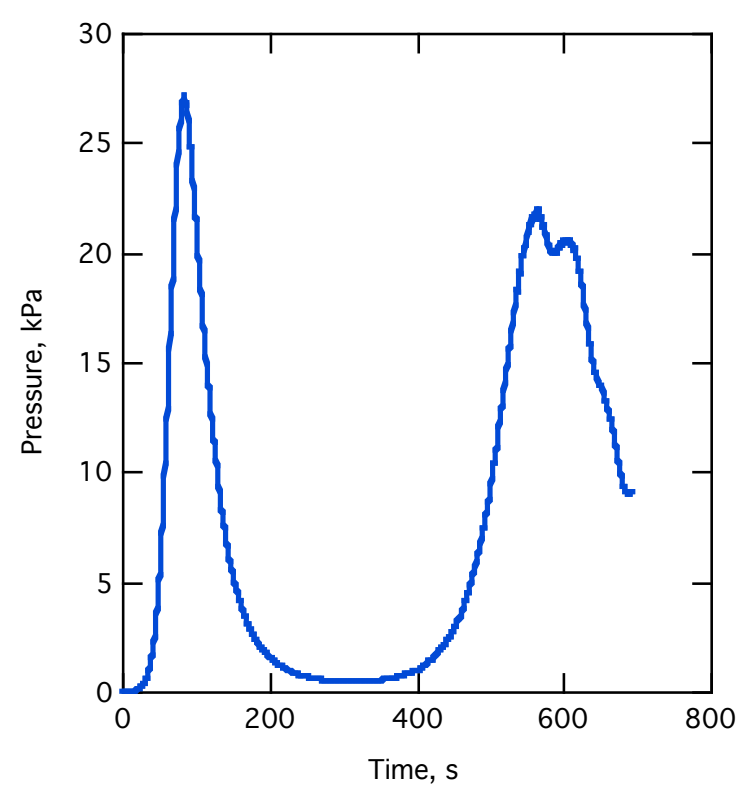

Figure 9. Pressure at a critical shoulder location on the Orion Crew Module.

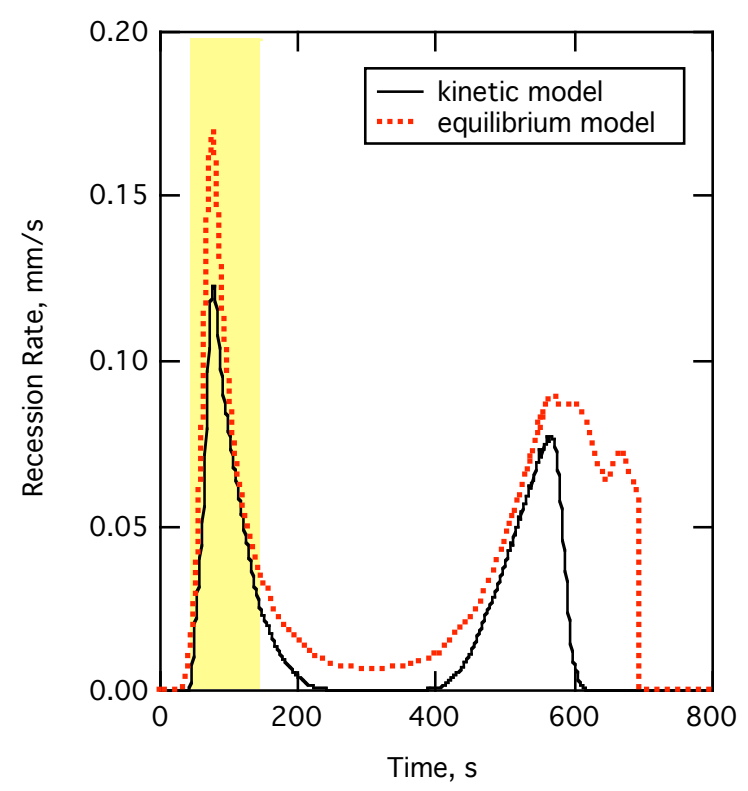

Figure 11. Recession rate predicted by ablation models for Orion environment.

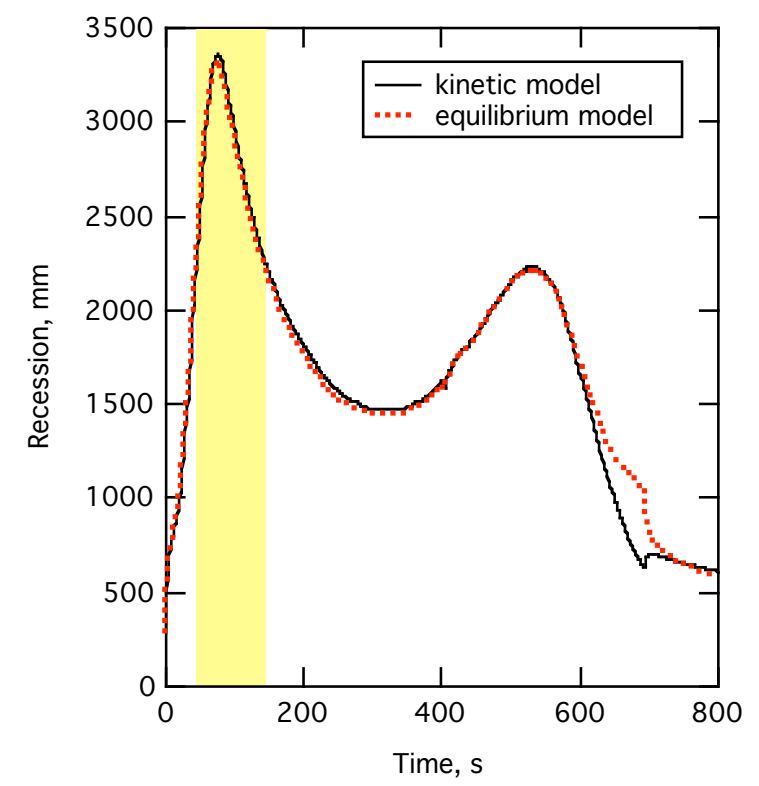

Figure 10. Surface temperature predicted by two ablation models for Orion environment. The equilibrium model is needed in the yellow-shaded region.

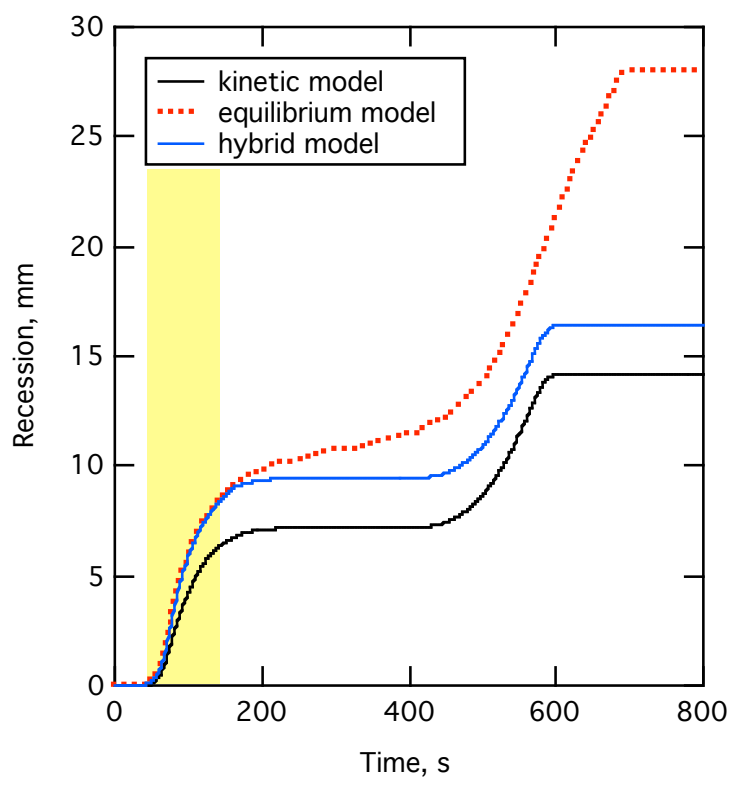

Figure 12. Total recession predicted by ablation models for Orion environment. 


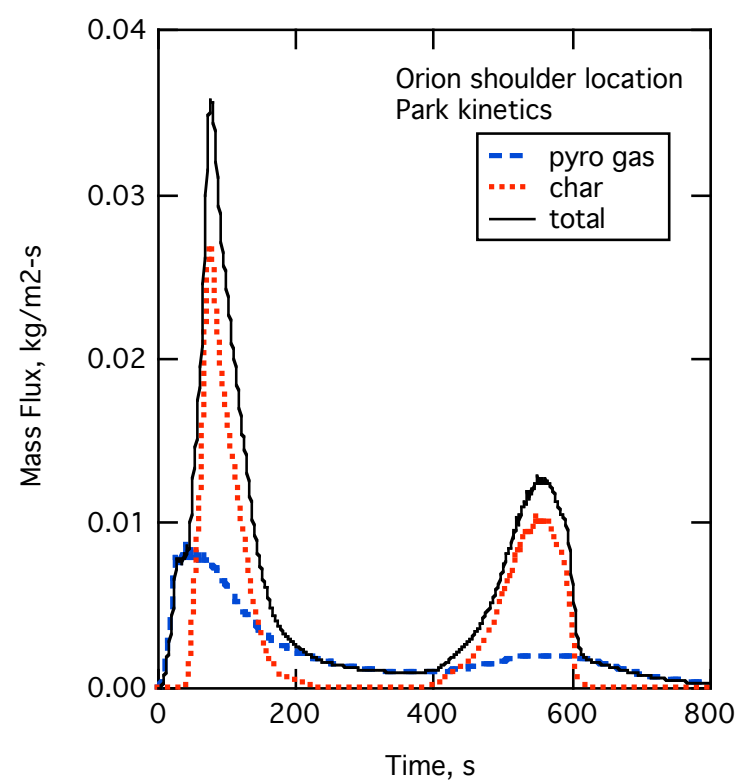

Figure 13. Surface blowing rates predicted by hybrid ablation model.
Plot to be inserted here

species vs time

Figure 14. Major blown species predicted by hybrid ablation model. 